PROCEEDINGS OF THE

AMERICAN MATHEMATICAL SOCIETY

Volume 131, Number 4, Pages 1053-1062

S 0002-9939(02)06634-0

Article electronically published on July 26, 2002

\title{
CATALAN PATHS AND QUASI-SYMMETRIC FUNCTIONS
}

\author{
J.-C. AVAL AND N. BERGERON
}

(Communicated by John R. Stembridge)

\begin{abstract}
We investigate the quotient ring $R$ of the ring of formal power series $\mathbb{Q}\left[\left[x_{1}, x_{2}, \ldots\right]\right]$ over the closure of the ideal generated by non-constant quasi-symmetric functions. We show that a Hilbert basis of the quotient is naturally indexed by Catalan paths (infinite Dyck paths). We also give a filtration of ideals related to Catalan paths from $(0,0)$ and above the line $y=x-k$. We investigate as well the quotient ring $R_{n}$ of polynomial ring in $n$ variables over the ideal generated by non-constant quasi-symmetric polynomials. We show that the dimension of $R_{n}$ is bounded above by the $n$th Catalan number.
\end{abstract}

\section{INTRODUCTION}

The ring Qsym of quasi-symmetric functions was introduced by Gessel [11] as a source of generating functions for $P$-partitions [16. Since then, quasi-symmetric functions have appeared in many combinatorial contexts [6, 16, 17]. The relation of Qsym to the ring of symmetric functions was first clarified by Malvenuto and Reutenauer [15] via a graded Hopf duality to the Solomon descent algebras, and then Gelfand et. al. [10] defined the graded Hopf algebra $N C$ of non-commutative symmetric functions and identified it with the Solomon descent algebra. In recent literature, we see a growing interest in quasi-symmetric functions and noncommutative symmetric functions as refinements of the ring of symmetric functions.

One unexplored avenue is as an analogue of the (symmetric) harmonic spaces. A classic combined result of Artin and Steinberg [1, 18, shows that the quotient ring of the polynomial ring $\mathbb{Q}\left[x_{1}, x_{2}, \ldots, x_{n}\right]$ in $n$ variables over the ideal $\mathcal{I}_{n}$ generated by non-constant symmetric polynomials has dimension $n$ !. In fact, this space is a graded symmetric group module that affords the left regular representation. Refinement and generalization of this result has lead to an explosion of incredible results and conjectures; for example see [4, 5, 7, 9, 12 for a small portion of this.

Here we are interested in the quotient ring $R$ of the ring of formal power series $\mathbb{Q}\left[\left[x_{1}, x_{2}, x_{3}, \ldots\right]\right]$ over the closure of the homogeneous ideal $\mathcal{J}$ generated by all non-constant quasi-symmetric functions. That is, the quotient

$$
R=\mathbb{Q}\left[\left[x_{1}, x_{2}, x_{3}, \ldots\right]\right] / \overline{\mathcal{J}} .
$$

To every monomial $x_{1}^{\tilde{\alpha}_{1}} x_{2}^{\tilde{\alpha}_{2}} x_{3}^{\tilde{\alpha}_{3}} \ldots$ (of finite total degree) in $\mathbb{Q}\left[\left[x_{1}, x_{2}, x_{3}, \ldots\right]\right]$ we associate a path in the plane as follows: $(0,0) \rightarrow\left(\tilde{\alpha}_{1}, 0\right) \rightarrow\left(\tilde{\alpha}_{1}, 1\right) \rightarrow\left(\tilde{\alpha}_{1}+\tilde{\alpha}_{2}, 1\right) \rightarrow$

Received by the editors October 16, 2001 and, in revised form, November 8, 2001.

2000 Mathematics Subject Classification. Primary 05E15, 05E10, 13P10, 13 F30.

The second author was supported in part by NSERC, PREA and CRC. 
$\left(\tilde{\alpha}_{1}+\tilde{\alpha}_{2}, 2\right) \rightarrow\left(\tilde{\alpha}_{1}+\tilde{\alpha}_{2}+\tilde{\alpha}_{3}, 2\right) \rightarrow \cdots$. If this path remains above the line $y=x$ we say that the path is a Catalan path (or infinite Dyck path). Our main result is

Theorem 1.1. A monomial Hilbert basis of $R$ is given by the monomials of $\mathbb{Q}\left[\left[x_{1}, x_{2}, x_{3}, \ldots\right]\right]$ corresponding to Catalan paths.

We also consider a special filtration of ideals $\mathcal{J}^{(e)}$ and their respective quotients, such that $\mathcal{J}=\mathcal{J}^{(0)}$ and $\mathcal{J}^{(e)} \subseteq \mathcal{J}^{(e+1)}$. The Hilbert basis of each quotient is indexed by paths above the line $y=x-e$.

Let $R_{n}$ denote the quotient of the polynomial ring $\mathbb{Q}\left[x_{1}, x_{2}, \ldots, x_{n}\right]$ over the ideal $\mathcal{J}_{n}$ generated by all non-constant quasi-symmetric polynomials. Recall that $C_{n}=\frac{1}{n+1}\left(\begin{array}{c}2 n \\ n\end{array}\right)$ are the famous Catalan numbers. The passage from infinitely many variables to finitely many variables is non-trivial and requires more work.

Theorem 1.2. $\operatorname{dim} R_{n} \leq C_{n}$.

In fact the equality holds in Theorem 1.2 and we have a complete proof of this together with François Bergeron [2], who has discovered the spaces $R_{n}$ completely independently and in the same period that we did. The results for $R_{n}$ are extremely interesting, and are the object of an ongoing collaboration with F. Bergeron and A. Garsia. We plan to write at least one more paper 3 to investigate further properties of $R_{n}$ and its generalization. In particular, the finite version of the successive quotients by the ideals $\mathcal{J}_{n}^{(e)}$ is related to the work of [5. Much of these results can be explained in a more general framework and will be the object of further study. We are convinced that these results are but the tip of a new iceberg. In particular, we would like to find any natural algebras acting on these spaces. What are the possible generalizations and specializations of the super-harmonics?

We underline here that F. Hivert [13] has developed an action of the Hecke algebra for which a polynomial is invariant if and only if it is quasi-symmetric. Unfortunately Hivert's action is not compatible with multiplication and does not preserve the ideal $\mathcal{J}_{n}$, hence it does not induce the desired action on the quotient. It is still interesting to note that Hivert's action is also related to Catalan numbers.

In Section 2 we recall appropriate definitions. In Section 3 we introduce a special family of generators for the ideal $\mathcal{J}$ and the associated filtration $\mathcal{J}^{(e)}$. In Section 4 we use these generators to show that the monomials corresponding to Catalan paths span our quotient, as well as the analogous result for $\mathcal{J}^{(e)}$. Theorem 1.2 follows from this section. To complete the proof of Theorem 1.1 we use a Gröbner basis argument in Section 5 to show independence.

\section{BASIC DEFINITIONS}

A composition $\alpha=\left[\alpha_{1}, \alpha_{2}, \ldots, \alpha_{k}\right]$ of a positive integer $d$ is an ordered list of positive integers whose sum is $d$. We denote this by $\alpha \models d$. We call the integers $\alpha_{i}$ the parts of $\alpha$, and denote the number of parts in $\alpha$ by $\ell(\alpha)$. Given two compositions $\alpha=\left[\alpha_{1}, \alpha_{2}, \ldots, \alpha_{k}\right]$ and $\beta=\left[\beta_{1}, \beta_{2}, \ldots, \beta_{\ell}\right]$, we denote by $\alpha \beta$ the concatenation product $\left[\alpha_{1}, \alpha_{2}, \ldots, \alpha_{k}, \beta_{1}, \beta_{2}, \ldots, \beta_{\ell}\right]$. Also, there exists a natural oneto-one correspondence between compositions of $d$ and subsets of $\{1,2, \ldots, d-1\}$. If $A=\left\{a_{1}, a_{2}, \ldots, a_{k-1}\right\} \subset[d-1]$, where $a_{1}<a_{2}<\ldots<a_{k-1}$, then $A$ corresponds to the composition, $\alpha=\left[a_{1}-a_{0}, a_{2}-a_{1}, \ldots, a_{k}-a_{k-1}\right]$, where $a_{0}=0$ and $a_{k}=d$. For ease of notation, we shall denote the set corresponding to a given composition $\alpha$ by $D(\alpha)$. For compositions $\alpha$ and $\beta$ we say that $\alpha$ is a refinement of $\beta$ if $D(\beta) \subset D(\alpha)$, and denote this by $\alpha \preccurlyeq \beta$. 
For any composition $\alpha=\left[\alpha_{1}, \alpha_{2}, \ldots, \alpha_{k}\right]$ of $d$ we denote by $M_{\alpha}$ the monomial quasi-symmetric function [11]: $M_{\alpha}\left(x_{1}, x_{2}, \ldots\right)=\sum_{i_{1}<i_{2}<\cdots<i_{k}} x_{i_{1}}^{\alpha_{1}} \ldots x_{i_{k}}^{\alpha_{k}}$. This is a homogeneous infinite series of degree $d$. We define $M_{0}=1$, where 0 denotes the unique empty composition of 0 . It is known from the work of Gessel that the monomial quasi-symmetric functions form a linear basis of a ring (in fact a Hopf algebra) Qsym of quasi-symmetric functions.

Another useful basis of the ring $Q s y m_{n}$ is given by the fundamental quasisymmetric function [11]:

$$
F_{\alpha}\left(x_{1}, x_{2}, \ldots\right)=\sum_{\alpha \succcurlyeq \beta} M_{\beta}\left(x_{1}, x_{2}, \ldots\right)=\sum_{\substack{j_{1} \leq j_{2} \leq \cdots \leq j_{d} \\ i \in D(\alpha) \nRightarrow j_{i}<j_{i+1}}} x_{j_{1}} x_{j_{2}} \cdots x_{j_{d}} .
$$

Fundamental quasi-symmetric functions satisfy the following obvious, but crucial, relations. For $\alpha=\left[\alpha_{1}, \alpha_{2}, \ldots, \alpha_{k}\right] \models d$,

$$
F_{\alpha}\left(x_{1}, x_{2}, \ldots\right)= \begin{cases}x_{1} F_{\left[\alpha_{1}-1, \alpha_{2}, \ldots, \alpha_{k}\right]}\left(x_{1}, x_{2}, \ldots\right)+F_{\alpha}\left(x_{2}, x_{3}, \ldots\right) & \text { if } \alpha_{1}>1, \\ x_{1} F_{\left[\alpha_{2}, \alpha_{3}, \ldots, \alpha_{k}\right]}\left(x_{2}, x_{3}, \ldots\right)+F_{\alpha}\left(x_{2}, x_{3}, \ldots\right) & \text { if } \alpha_{1}=1 .\end{cases}
$$

Here $F_{\alpha}\left(x_{2}, x_{3}, \ldots\right)$ is the function $F_{\alpha}\left(x_{1}, x_{2}, \ldots\right)$ in which the variable $x_{i}$ is replaced by $x_{i+1}$. These relations are the key ingredients in our proof.

In the following we have to consider generalized (infinite) compositions. That is, a sequence $\tilde{\alpha}=\left(\tilde{\alpha}_{1}, \tilde{\alpha}_{2}, \ldots\right)$ such that the parts $\tilde{\alpha}_{j} \geq 0$ for $j \geq 1$ (we allow some parts to be zero) and the sum of the parts $d(\tilde{\alpha})=\sum \tilde{\alpha}_{i}<\infty$. We say that $\tilde{\alpha}$ is a generalized composition of $d(\tilde{\alpha})<\infty$. We use a " $"$ to indicate that we have a generalized composition and no " " if the composition is standard, that is, without zeros. We also consider generalized compositions of finite length and denote by $\ell(\tilde{\alpha})$ the number of parts of $\tilde{\alpha}$. The concatenation of a finite length generalized composition $\tilde{\alpha}$ with an infinite one $\tilde{\beta}$ is denoted by $\tilde{\alpha} \tilde{\beta}$. We also write $\tilde{\alpha}+\tilde{\beta}$ and $\tilde{\alpha} \leq \tilde{\beta}$ to denote the componentwise sum and componentwise inequalities, respectively. For an infinite generalized composition $\tilde{\alpha}$, since $d(\tilde{\alpha})<\infty$, only finitely many parts of $\tilde{\alpha}$ are non-zero. Thus there is always a finite generalized composition $\tilde{\nu}$ such that $\tilde{\alpha}=\tilde{\nu} 00 \cdots$.

In this paper, we devote our attention to the ideal $\mathcal{J}=\left\langle F_{\alpha}\left(x_{1}, x_{2}, \ldots\right)\right\rangle_{\alpha \models d>0}$ of $\mathbb{Q}\left[\left[x_{1}, x_{2}, \ldots\right]\right]$ generated by the non-constant quasi-symmetric functions, and consider the quotient

$$
R=\mathbb{Q}\left[\left[x_{1}, x_{2}, \ldots\right]\right] / \overline{\mathcal{J}},
$$

where $\overline{\mathcal{J}}$ denotes the closure (with respect to the standard topology with formal power series) of $\mathcal{J}$ in $\mathbb{Q}\left[\left[x_{1}, x_{2}, \ldots\right]\right]$.

\section{The generators $G_{\tilde{\alpha}}$}

We exploit the relation (2.1) to construct a set $\left\{G_{\tilde{\alpha}}\right\} \subseteq \mathcal{J}$ indexed by the generalized (infinite) composition $\tilde{\alpha}$ such that there exists a factorization $\tilde{\alpha}=\tilde{\pi} \tilde{\rho}$ where $d(\tilde{\pi})-\ell(\tilde{\pi}) \geq 0$. We first define recursively the functions $G_{\tilde{\alpha}}$ for all infinite generalized composition $\tilde{\alpha}$. Then in Lemma 3.1 we characterize the $\tilde{\alpha}$ obtained from the transitive closure of the $G_{\tilde{\alpha}} \in \mathcal{J}$. Let $\tilde{\alpha}=\tilde{\nu} 00 \cdots$ where $\ell(\tilde{\nu})<\infty$ and the last part of $\tilde{\nu}$ is non-zero, or $\tilde{\alpha}=00 \cdots$. Our definition is recursive on $n=\ell(\tilde{\nu})$. If 
$\tilde{\nu}=\nu$ is a standard composition, then let

$$
G_{\tilde{\alpha}}=F_{\nu}\left(x_{1}, x_{2}, \ldots\right) .
$$

If $\ell(\tilde{\nu})=0$, then this formula gives $G_{00} \ldots=1$. Assume now that $\tilde{\nu}$ is non-standard and let $\tilde{\nu}=\tilde{\gamma} 0 a \beta$ be the unique factorization of $\tilde{\nu}$ such that $a>0$ is a positive integer, $\beta$ is a (possibly empty) standard composition and $\tilde{\gamma}$ is a (possibly empty) generalized composition. For $\tilde{\alpha}=\tilde{\gamma} 0 a \beta 00 \cdots$ and $k=\ell(\tilde{\gamma} 0)=\ell(\tilde{\gamma})+1$, we define

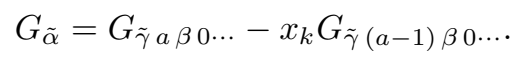

Both terms on the right are well defined by induction since $\ell(\tilde{\gamma} a \beta)=\ell(\tilde{\nu})-1<n$.

We now characterize the transitive closure of the definition (3.1) and (3.2) within $\mathcal{J}$. At this point it is useful to introduce the following family of ideals. For any $e \geq$ 0 , let $\mathcal{J}^{(e)}=\left\langle F_{\alpha}: \exists \pi \rho=\alpha, d(\pi)-\ell(\pi) \geq e\right\rangle$. This is a filtration $\mathcal{J}^{(e)} \subseteq \mathcal{J}^{(e+1)}$ such that $\mathcal{J}=\mathcal{J}^{(0)}$. For a generalized composition $\tilde{\alpha}$, we say that it reaches level $e$ if there exists a factorization $\tilde{\alpha}=\tilde{\pi} \tilde{\rho}$ such that $d(\tilde{\pi})-\ell(\tilde{\pi}) \geq e$.

\section{Lemma 3.1.}

1. If $\tilde{\alpha}$ reaches level $e$, then $G_{\tilde{\alpha}} \in \mathcal{J}^{(e)}$.

2. Conversely, in (3.2), if $\tilde{\gamma}(a-1) \beta 0 \cdots$ reaches level e, then $\tilde{\alpha}$ reaches level e.

Proof. For the first statement we proceed by induction on $\ell(\tilde{\nu})$ where $\tilde{\alpha}=\tilde{\nu} 00 \cdots$ and the last part of $\tilde{\nu}$ is non-zero. If $\ell(\tilde{\nu})=0$, then $G_{00 \cdots}=1$ is not in any of the ideals $\mathcal{J}^{(e)}$. Assume that $\ell(\tilde{\nu})>0$. We first consider the case when $\tilde{\nu}=\nu$ is a standard composition. If $\tilde{\alpha}$ reaches level $e$, then so is $\nu$ and we have $G_{\tilde{\alpha}}=$ $F_{\nu}\left(x_{1}, x_{2}, \ldots\right) \in \mathcal{J}^{(e)}$. If $\tilde{\nu}$ is a non-standard generalized composition, then let $\tilde{\alpha}=\tilde{\pi} \tilde{\rho}$ be the factorization such that $d(\tilde{\pi})-\ell(\tilde{\pi}) \geq e$, and let $\tilde{\alpha}=\tilde{\nu} 00 \cdots=$ $\tilde{\gamma} 0 a \beta 00 \cdots$ be the factorization used in (3.2). If $\tilde{\pi}$ is an initial factor of $\tilde{\gamma}$, then it is clearly an initial factor of both $\tilde{\gamma} a \beta 00 \cdots$ and $\tilde{\gamma}(a-1) \beta 00 \cdots$ and they both reach level $e$. By the induction hypothesis, both $G_{\tilde{\gamma} a \beta 0} \ldots$ and $G_{\tilde{\gamma}(a-1) \beta 0 \ldots}$ are in $\mathcal{J}^{(e)}$ and in turn $G_{\tilde{\alpha}} \in \mathcal{J}^{(e)}$. If we now assume that $\tilde{\pi}=\tilde{\gamma} 0$, then $d(\tilde{\gamma})-\ell(\tilde{\gamma})=$ $d(\tilde{\pi})-(\ell(\tilde{\pi})-1) \geq e+1>e$ and again the induction hypothesis can be applied to (3.2) to show that $G_{\tilde{\alpha}} \in \mathcal{J}^{(e)}$.

We are left to check the case where $\tilde{\pi}=\tilde{\gamma} 0 a \tilde{\mu}$. For the first term in (3.2), $\tilde{\gamma} a \tilde{\mu}$ is an initial factor and $d(\tilde{\mu} a \tilde{\gamma})-\ell(\tilde{\gamma} a \tilde{\mu})=d(\tilde{\pi})-(\ell(\tilde{\pi})-1) \geq e+1>e$. The

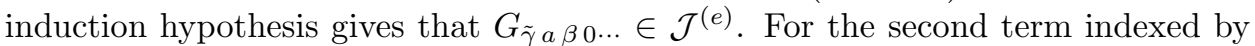
$\tilde{\gamma}(a-1) \beta 00 \cdots$ we have $d(\tilde{\gamma}(a-1) \tilde{\mu})-\ell(\tilde{\gamma}(a-1) \tilde{\mu})=(d(\tilde{\pi})-1)-(\ell(\tilde{\pi})-1) \geq e$. Again the induction hypothesis gives us that $G_{\tilde{\gamma}(a-1)} \beta 0 \ldots \in \mathcal{J}^{(e)}$, concluding the proof that $G_{\tilde{\alpha}} \in \mathcal{J}^{(e)}$.

For the second statement of the lemma let $\tilde{\gamma}(a-1) \beta 00 \cdots=\tilde{\pi} \tilde{\rho}$ be a factorization such that $d(\tilde{\pi})-\ell(\tilde{\pi}) \geq e$. If $\tilde{\pi}$ is an initial factor of $\tilde{\gamma}$, then it is clear that $\tilde{\alpha}$ reaches level $e$. On the other hand if $\tilde{\pi}=\tilde{\gamma}(a-1) \tilde{\mu}$, then we have $d(\tilde{\gamma} 0 a \tilde{\mu})-\ell(\tilde{\gamma} 0 a \tilde{\mu})=(d(\tilde{\gamma}(a-1) \tilde{\mu})+1)-(\ell(\tilde{\gamma}(a-1) \tilde{\mu})+1) \geq e$. Thus $\tilde{\alpha}$ reaches level $e$.

In light of the previous lemma, let $\mathcal{G}^{(e)}$ denote the set of all generalized infinite compositions $\tilde{\alpha}$ reaching level $e$, that satisfy $d(\tilde{\pi})-\ell(\tilde{\pi}) \geq e$. We remark that the set $\left\{G_{\tilde{\alpha}}\right\}_{\tilde{\alpha} \in \mathcal{G}^{(e)}}$ constructed above is contained in $\mathcal{J}^{(e)}$ and contains $\left\{F_{\alpha}\left(x_{1}, x_{2}, \ldots\right)\right.$ : $\exists \alpha=\pi \rho, d(\pi)-\ell(\pi) \geq e\}$. Hence we have 
Lemma 3.2. $\mathcal{J}^{(e)}=\left\langle G_{\tilde{\alpha}}\right\rangle_{\tilde{\alpha} \in \mathcal{G}^{(e)}}$.

Our next task is to characterize the leading monomial of each function $G_{\tilde{\alpha}}$. Before this we need to specify which monomial order we use. Let $X^{\tilde{\alpha}}=x_{1}^{\tilde{\alpha}_{1}} x_{2}^{\tilde{\alpha}_{2}} \ldots$ and $X^{\tilde{\beta}}=x_{1}^{\tilde{\beta}_{1}} x_{2}^{\tilde{\beta}_{2}} \cdots$ be any two monomials where $\tilde{\alpha}$ and $\tilde{\beta}$ are two generalized infinite compositions. We say that $X^{\tilde{\alpha}} \leq_{l e x} X^{\tilde{\beta}}$ if and only if $d(\tilde{\alpha})>d(\tilde{\beta})$, or $d(\tilde{\alpha})=d(\tilde{\beta})$ and the leftmost non-zero entry in $\left[\tilde{\beta}_{1}-\tilde{\alpha}_{1}, \tilde{\beta}_{2}-\tilde{\alpha}_{2}, \ldots,\right]$ is positive. The order $\leq_{l e x}$ is a classical monomial order in the sense that it is a total order and if $X^{\tilde{\alpha}} \leq_{\text {lex }} X^{\tilde{\beta}}$, then $X^{\tilde{\alpha}} X^{\tilde{\gamma}}=X^{\tilde{\alpha}+\tilde{\gamma}} \leq_{\text {lex }} X^{\tilde{\beta}+\tilde{\gamma}}=X^{\tilde{\beta}} X^{\tilde{\gamma}}$. Here the sum of generalized compositions is componentwise.

For any formal power series $P=P\left(x_{1}, x_{2}, \ldots\right) \in \mathbb{Q}\left[\left[x_{1}, x_{2}, \ldots\right]\right]$ we let $L M(P)$ denote the leading monomial of $P$. That is, $L M(P)$ is the monomial of $P$ with nonzero coefficient of smallest degree and largest in lexicographic order. In other words, the leading monomial for the order $\leq_{l e x}$. We let $L C(P)$ denote the coefficient of $L M(P)$ in $P$. Remark that for any two functions $P$ and $Q$, we have $L M(P Q)=$ $\operatorname{LM}(P) L M(Q)$. We need the following result which is the extension for the $G$ functions of relation (2.1) for the $F$-functions.

Lemma 3.3. Let $\tilde{\alpha}=b \tilde{\rho}$ be any generalized infinite composition and $b \geq 0$.

$$
G_{\tilde{\alpha}}\left(x_{1}, x_{2}, \ldots\right)= \begin{cases}G_{\tilde{\rho}}\left(x_{2}, x_{3}, \ldots\right) & \text { if } b=0, \\ x_{1} G_{(b-1) \tilde{\rho}}+R_{\tilde{\alpha}}\left(x_{2}, x_{3}, \ldots\right) & \text { if } b>0 .\end{cases}
$$

Proof. We remark that the function $G_{\tilde{\rho}}\left(x_{2}, x_{3}, \ldots\right)$ is the function $G_{\tilde{\rho}}\left(x_{1}, x_{2}, \ldots\right)$ in which the variable $x_{i}$ is replaced by $x_{i+1}$. We let $\tilde{\alpha}=\tilde{\nu} 00 \cdots$ and proceed by induction on $\ell(\tilde{\nu})$. If $\ell(\tilde{\nu})=0$, then we have $G_{00 \ldots}=1$ and $(3.3)$ is valid. In the following we extensively use the relation (2.1). If $\tilde{\alpha}=b \rho 00 \cdots$, then (3.1) gives us $G_{\tilde{\alpha}}=F_{b \rho}\left(x_{1}, x_{2}, \ldots\right)$. If $b>1$, then we get

$$
\begin{aligned}
G_{b \rho 0 \ldots} & =F_{b \rho}\left(x_{1}, x_{2}, \ldots\right)=x_{1} F_{(b-1) \rho}\left(x_{1}, x_{2}, \ldots\right)+F_{b \rho}\left(x_{2}, x_{3}, \ldots\right) \\
& =x_{1} G_{(b-1) \rho 0 \ldots}+R_{b \rho 0 \ldots}\left(x_{2}, x_{3}, \ldots\right),
\end{aligned}
$$

and (3.3) follows for this case with $R_{\tilde{\alpha}}\left(x_{2}, x_{3}, \ldots\right)=F_{b \rho}\left(x_{2}, x_{3}, \ldots\right)$.

For $b=1$, we first need to understand (3.3) in the case $G_{0} \rho 0 \ldots$ For this assume that $\rho=a \beta$. If $a>1$, then the definitions (3.1) and (3.2) give

$$
\begin{aligned}
G_{0 a \beta 0 \cdots} & =G_{a \beta 0 \ldots-x_{1}} G_{(a-1) \beta 0 \ldots}=\left(F_{a \beta}-x_{1} F_{(a-1) \beta}\right)\left(x_{1}, x_{2}, \ldots\right) \\
& =F_{a \beta}\left(x_{2}, x_{3}, \ldots\right)=G_{a \beta 0 \cdots}\left(x_{2}, x_{3}, \ldots\right) .
\end{aligned}
$$

If $a=1$, then we use the induction hypothesis on $\ell(0 \beta)=\ell(01 \beta)-1<\ell(\tilde{\nu})$ to get

$$
\begin{aligned}
& G_{01 \beta 0 \ldots}=G_{1 \beta 0 \ldots}-x_{1} G_{0 \beta 0 \ldots}=F_{1 \beta}\left(x_{1}, x_{2}, \ldots\right)-x_{1} F_{\beta}\left(x_{2}, x_{3}, \ldots\right) \\
& =F_{1 \beta}\left(x_{2}, x_{3}, \ldots\right)=G_{1 \beta 0 \ldots}\left(x_{2}, x_{3}, \ldots\right) \text {. }
\end{aligned}
$$

Now we can go back to the case of $\tilde{\alpha}=1 \rho 0 \cdots$ :

$$
\begin{aligned}
G_{1 \rho 0 \ldots} & =F_{1 \rho}\left(x_{1}, x_{2}, \ldots\right)=x_{1} F_{\rho}\left(x_{2}, x_{3}, \ldots\right)+F_{1 \rho}\left(x_{2}, x_{3}, \ldots\right) \\
& =x_{1} G_{0 \rho 0 \ldots}+R_{1 \rho 0 \ldots}\left(x_{2}, x_{3}, \ldots\right),
\end{aligned}
$$

where $R_{1 \rho 0 \ldots}\left(x_{2}, x_{3}, \ldots\right)=F_{1 \rho}\left(x_{2}, x_{3}, \ldots\right)$.

We then consider when $\tilde{\alpha}=\tilde{\gamma} 0 a \beta 00 \cdots$. This is the factorization needed to use (3.2) with $k=\ell(\tilde{\gamma})+1$. If $\tilde{\gamma}$ is empty, then we have $b=0$ and we are in the case considered above. Assume that $\tilde{\gamma}=b \tilde{\mu}$. If $b=0$, then applying the induction 
hypothesis we have

$$
\begin{aligned}
& \left.\left.G_{0 \tilde{\mu} 0 a \beta 0 \ldots\left(x_{1}\right.}, x_{2}, \ldots\right)=G_{0 \tilde{\mu} a \beta 0 \ldots}\left(x_{1}, x_{2}, \ldots\right)-x_{k} G_{0 \tilde{\mu}(a-1) \beta 0 \ldots\left(x_{1}\right.}, x_{2}, \ldots\right) \\
& =G_{\tilde{\mu} a \beta 0 \ldots}\left(x_{2}, x_{3}, \ldots\right)-x_{(k-1)+1} G_{\tilde{\mu}(a-1) \beta 0 \ldots}\left(x_{2}, x_{3}, \ldots\right) \\
& =G_{\tilde{\mu} 0 a \beta 0 \ldots\left(x_{2}, x_{3}, \ldots\right) .}
\end{aligned}
$$

Here we remark that even though $\ell(\tilde{\mu})+1=k-1$, we have to replace $x_{k-1}$ by $x_{(k-1)+1}=x_{k}$ in the recurrence for $G_{\tilde{\mu} 0 a \beta 0 \ldots}\left(x_{2}, x_{3}, \ldots\right)$. For $b>0$,

$$
\begin{aligned}
& G_{b \tilde{\mu} 0 a \beta 0 \cdots}=G_{b \tilde{\mu} a \beta 0 \ldots-x_{k}} G_{b \tilde{\mu}(a-1) \beta 0 \cdots}
\end{aligned}
$$

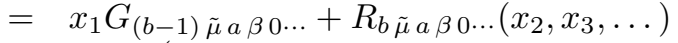

$$
\begin{aligned}
& -x_{k}\left(x_{1} G_{(b-1) \tilde{\mu}(a-1) \beta 0 \ldots}+R_{b \tilde{\mu}(a-1) \beta 0 \ldots}\left(x_{2}, x_{3}, \ldots\right)\right)
\end{aligned}
$$

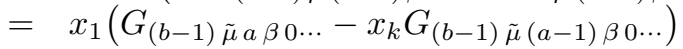

$$
\begin{aligned}
& +R_{b \tilde{\mu} a \beta 0 \ldots}\left(x_{2}, x_{3}, \ldots\right)-x_{k} R_{b \tilde{\mu}(a-1) \beta 0 \ldots\left(x_{2}, x_{3}, \ldots\right)}
\end{aligned}
$$

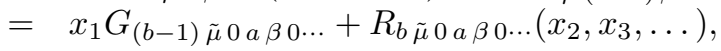

where $R_{b \tilde{\mu} 0 a \beta 0 \ldots}\left(x_{2}, x_{3}, \ldots\right)=\left(R_{b \tilde{\mu} a \beta 0 \ldots}-x_{k} R_{b \tilde{\mu}(a-1) \beta 0 \ldots}\right)\left(x_{2}, x_{3}, \ldots\right)$.

Corollary 3.4. For any generalized composition $\tilde{\alpha}$ we have $L M\left(G_{\tilde{\alpha}}\right)=X^{\tilde{\alpha}}$.

Proof. Let $\tilde{\alpha}=\tilde{\nu} 00 \cdots$. We proceed by induction on $\ell(\tilde{\nu})$ and the degree $d=$ $d(\tilde{\alpha})=\sum \tilde{\alpha}_{i}$. If $\ell(\tilde{\nu})=0$ we have $G_{00 \ldots}=1=X^{00 \cdots}$. If $\ell(\tilde{\nu}) \geq 1$, then let $\tilde{\alpha}=b \tilde{\rho}$ as in Lemma 3.3 . If $b=0$, then the induction hypothesis on $\ell(\tilde{\nu})$ gives $\operatorname{LM}\left(G_{0} \tilde{\rho}\right)=\operatorname{LM}\left(G_{\tilde{\rho}}\left(x_{2}, x_{3}, \ldots\right)\right)=x_{1}^{0} x_{2}^{\tilde{\rho}_{1}} x_{3}^{\tilde{\rho}_{2}} \cdots=X^{\tilde{\alpha}}$. Now if $b>0$ we use the second part of Lemma 3.3 and the induction hypothesis on $d$, and get $L M\left(x_{1} G_{(b-1) \tilde{\mu}}+R_{\tilde{\alpha}}\left(x_{2}, x_{3}, \ldots\right)\right)=x_{1} L M\left(G_{(b-1) \tilde{\mu}}\right)=X^{\tilde{\alpha}}$.

Remark 3.5. From the above corollary, by triangularity, it is clear that the set $\left\{G_{\tilde{\alpha}}\right\}$ for all $\tilde{\alpha}$ forms a Hilbert basis of $\mathbb{Q}\left[\left[x_{1}, x_{2} \ldots\right]\right]$. We will see in Section 5 that in fact $\left\{G_{\tilde{\alpha}}\right\}_{\tilde{\alpha} \in \mathcal{G}^{(e)}}$ forms a Hilbert basis of $\overline{\mathcal{J}^{(e)}}$.

\section{It is at most Catalan}

Let $\mathcal{Q}^{(e)}=\left\{G_{\tilde{\alpha}}\right\}_{\tilde{\alpha} \in \mathcal{G}^{(e)}}$ be the generating set of $\mathcal{J}^{(e)}$ constructed in Lemma 3.2 In this section we show that after reduction, at most the monomials corresponding to Catalan paths form a Hilbert basis of $R=\mathbb{Q}\left[\left[x_{1}, x_{2}, \ldots\right]\right] / \overline{\mathcal{J}}$. For this we reduce every other monomial to these. In fact for $R^{(e)}=\mathbb{Q}\left[\left[x_{1}, x_{2}, \ldots\right]\right] / \overline{\mathcal{J}^{(e)}}$, we show that at most the monomials corresponding to paths above the line $y=x-e$ form a Hilbert basis of $R^{(e)}$, for all $e \geq 0$. We conclude this section with the corresponding result for finitely many variables, $R_{n}^{(e)}=\mathbb{Q}\left[x_{1}, x_{2}, \ldots, x_{n}\right] / \mathcal{J}_{n}^{(e)}$, which is a generalization of Theorem 1.2.

Given any generalized infinite composition $\tilde{\alpha}$ we associate a unique path in the plane with steps going north or east. More precisely, for $\tilde{\alpha}=\left(\tilde{\alpha}_{1}, \tilde{\alpha}_{2}, \tilde{\alpha}_{3}, \ldots\right)$, we construct the path $(0,0) \rightarrow\left(\tilde{\alpha}_{1}, 0\right) \rightarrow\left(\tilde{\alpha}_{1}, 1\right) \rightarrow\left(\tilde{\alpha}_{1}+\tilde{\alpha}_{2}, 1\right) \rightarrow\left(\tilde{\alpha}_{1}+\tilde{\alpha}_{2}, 2\right) \rightarrow$ $\left(\tilde{\alpha}_{1}+\tilde{\alpha}_{2}+\tilde{\alpha}_{3}, 2\right) \rightarrow \cdots$. For example, for $\tilde{\alpha}=0210300 \cdots$ we have the path

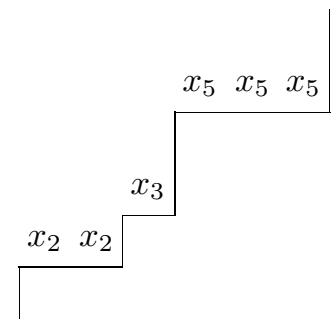


For every east step at height $i-1$ we associate a variable $x_{i}$. The product of all the variables associated to a path encoded by $\tilde{\alpha}$ is denoted $X^{\tilde{\alpha}}$. We now remark that for any factorization $\tilde{\alpha}=\tilde{\pi} \tilde{\rho}$, the rightmost coordinate of the path at height $\ell(\tilde{\pi})-1$ is $(d(\tilde{\pi}), \ell(\tilde{\pi})-1)$.

Definition 4.1. For an integer $e \geq 0$, we say that a generalized composition $\tilde{\alpha}$ is of type $e$-Catalan if its associated path remains above the line $y=x-e$. That is, every coordinate $\left(x_{i}, y_{i}\right)$ of the path is such that $x_{i}-y_{i} \leq e$.

Lemma 4.2. The monomials of $\mathbb{Q}\left[\left[x_{1}, x_{2}, \ldots\right]\right]$ corresponding to paths remaining above the line $y=x-e$ contain a Hilbert basis of the quotient $R^{(e)}$.

Proof. Let $X^{\tilde{\alpha}}$ be any monomial of degree $d$. If the path corresponding to $\tilde{\alpha}$ goes under the line $y=x-e$, then let $\tilde{\alpha}=\tilde{\pi} \tilde{\rho}$ be any factorization such that the coordinate $(d(\tilde{\pi}), \ell(\tilde{\pi})-1)$ is under the line $y=x-e$. That is, $d(\tilde{\pi})-\ell(\tilde{\pi}) \geq e$. From Lemma 3.1 we conclude that the function $G_{\tilde{\alpha}}$ with leading monomial $X^{\tilde{\alpha}}$ is in $\mathcal{J}^{(e)}$. This monomial can thus be replaced by monomials of degree $d$ but strictly smaller with respect to $<_{l e x}$. Repeating this step (possibly countably many times) with the next largest monomial going under the line $y=x-e$, any monomial $X^{\tilde{\alpha}}$ can be reduced modulo the ideal $\overline{\mathcal{J}^{(e)}}$ to a series containing only monomials $X^{\tilde{\beta}}$ where $\tilde{\beta}$ is of type $e$-Catalan.

We are now in a position to generalize Theorem 1.2 and prove it. For this, note that the quasi-symmetric polynomials in $n$ variables are defined by setting $0=$ $x_{n+1}=x_{n+2}=\cdots$ in the quasi-symmetric functions. That is, $F_{\alpha}\left(x_{1}, x_{2}, \ldots, x_{n}\right)=$ $F_{\alpha}\left(x_{1}, x_{2}, \ldots, x_{n}, 0,0, \ldots\right)$. We then define $J_{n}^{(e)}=\left\langle F_{\alpha}\left(x_{1}, x_{2}, \ldots, x_{n}\right): \alpha\right.$ reaches level $e\rangle$ and $R_{n}^{(e)}=\mathbb{Q}\left[x_{1}, x_{2}, \ldots, x_{n}\right] / J_{n}^{(e)}$. Similarly we set $G_{\tilde{\alpha}}\left(x_{1}, x_{2}, \ldots, x_{n}\right)=$ $G_{\tilde{\alpha}}\left(x_{1}, x_{2}, \ldots, x_{n}, 0,0, \ldots\right)$. It is clear that Lemma 3.1 holds for $J_{n}^{(e)}$ in the same way. More over if $\tilde{\alpha}=\tilde{\nu} 00 \cdots$ for $\ell(\tilde{\nu})=n$, then

$$
L M\left(G_{\tilde{\alpha}}\left(x_{1}, x_{2}, \ldots, x_{n}\right)\right)=L M\left(G_{\tilde{\alpha}}\left(x_{1}, x_{2}, \ldots, x_{n}, 0,0, \ldots\right)\right)=x_{1}^{\tilde{\nu}_{1}} x_{2}^{\tilde{\nu}_{2}} \cdots x_{n}^{\tilde{\nu}_{n}} .
$$

Let $C_{n}^{(e)}$ denote the number of generalized compositions $\tilde{\alpha}$ of type $e$-Catalan such that $\tilde{\alpha}=\tilde{\nu} 00 \cdots$ and $\ell(\tilde{\nu})=n$. These are in bijection with the paths from $(0,0)$ to $(n+e, n)$ that remain above the line $y=x-e$. Indeed, if we have a path of type $e$-Catalan, it suffices to add a horizontal line from $\left(\tilde{\nu}_{1}+\tilde{\nu}_{2}+\cdots+\tilde{\nu}_{n}, n\right)$ to $(n+e, n)$. When $e=0$, we have $C_{n}^{(0)}=C_{n}$, the $n$th Catalan number. This enumerates the classical Dyck paths from $(0,0)$ to $(n, n)$ remaining above the line $y=x$. See [16] for an extensive account on Catalan numbers. We have the following generalization to our Theorem 1.2.

Corollary 4.3. $\operatorname{dim}\left(R_{n}^{(e)}\right) \leq C_{n}^{(e)}$.

Proof. We use the same argument as in Lemma 4.2. For this we use the fact that for any monomial $x_{1}^{\tilde{\nu}_{1}} x_{2}^{\tilde{\nu}_{2}} \cdots x_{n}^{\tilde{\nu}_{n}}$ in $\mathbb{Q}\left[x_{1}, x_{2}, \ldots, x_{n}\right]$, if $\tilde{\nu}$ reaches level $e$, then $G_{\tilde{\nu} 0 \ldots}\left(x_{1}, x_{2}, \ldots, x_{n}\right) \in \mathcal{J}_{n}^{(e)}$. Hence a basis of $R_{n}^{(e)}$ is contained in the monomials corresponding to paths of type $e$-Catalan and our result follows.

Again the equality holds and we address this question in [2]. 


\section{It is A HiLberT BASIS}

In the previous section, the generating set $\mathcal{Q}^{(e)}=\left\{G_{\tilde{\alpha}}\right\}_{\tilde{\alpha} \in \mathcal{G}^{(e)}}$ of $\mathcal{J}^{(e)}$ was used to reduce every monomial to $e$-Catalan type generalized compositions. It is in fact a Hilbert basis for the given ideal. We use here ideas of Gröbner basis theory on valuation rings. This is crucial to complete the proof of Theorem 1.1 Let us recall a few basic facts about Gröbner bases; see 8] for more details.

To show that a set $S$ is a Gröbner basis it is enough to show that all polynomial syzygies of that set are reducible in $S$. The polynomial syzygy of $P$ and $Q$ is defined by $S(P, Q)=L C(Q) M_{1} P-L C(P) M_{2} Q$ where $\operatorname{lcm}(L M(P), L M(Q))=$ $M_{1} \cdot L M(P)=M_{2} \cdot L M(Q)$. This shows that the given set contains all the generators of the leading monomials of the ideal. We use the classic Buchberger's lemma [8]:

Lemma 5.1. Assume $P, Q \in S$. If there is an $R \in S$ such that $L M(R)$ divides lcm $(L M(P), L M(Q))$, and if both $S(R, Q)$ and $S(R, P)$ are reducible in $S$, then $S(P, Q)$ is reducible in $S$.

This result is easily adapted to our context. We first remark that our sets $\mathcal{Q}^{(e)}$ satisfy the following useful property:

Lemma 5.2. For all $\tilde{\alpha} \leq \tilde{\rho}$ (componentwise),

$$
G_{\tilde{\alpha}} \in \mathcal{Q}^{(e)} \quad \Longrightarrow \quad G_{\tilde{\rho}} \in \mathcal{Q}^{(e)} .
$$

Proof. If $\tilde{\alpha}=\tilde{\pi} \tilde{\nu}$ satisfies $d(\tilde{\pi})-\ell(\tilde{\pi}) \geq e$, then let $r=\ell(\tilde{\pi})$ and consider $\tilde{\rho}=\tilde{\gamma} \tilde{\mu}$ where $\ell(\tilde{\gamma})=r$. Since $d(\tilde{\pi}) \leq d(\tilde{\gamma})$, we have $d(\tilde{\gamma})-\ell(\tilde{\gamma}) \geq d(\tilde{\pi})-\ell(\tilde{\pi}) \geq e$. By Lemma 3.1, $G_{\tilde{\rho}} \in \mathcal{Q}^{(e)}$.

We can now adapt the proof (see [8]) of Lemma 5.1 to our situation. For any pair $\tilde{\alpha}, \tilde{\pi} \in \mathcal{G}^{(e)}$, we define $S\left(G_{\tilde{\alpha}}, G_{\tilde{\pi}}\right)$ as before with our definition of $L M$ and $L C$. We show in this section that any such $S\left(G_{\tilde{\alpha}}, G_{\tilde{\pi}}\right)$ is reducible in $\mathcal{Q}^{(e)}$. Let $\tilde{\rho} \in \mathcal{G}^{(e)}$ be the unique element such that $X^{\tilde{\rho}}=\operatorname{lcm}\left(X^{\tilde{\alpha}}, X^{\tilde{\pi}}\right)=M_{1} X^{\tilde{\alpha}}=M_{2} X^{\tilde{\alpha}}$. We have

$$
\begin{aligned}
S\left(G_{\tilde{\alpha}}, G_{\tilde{\pi}}\right) & =M_{1} G_{\tilde{\alpha}}-M_{2} G_{\tilde{\pi}}=M_{1} G_{\tilde{\alpha}}-G_{\tilde{\rho}}+G_{\tilde{\rho}}-M_{2} G_{\tilde{\pi}} \\
& =S\left(G_{\tilde{\alpha}}, G_{\tilde{\rho}}\right)+S\left(G_{\tilde{\rho}}, G_{\tilde{\pi}}\right) .
\end{aligned}
$$

If both $S\left(G_{\tilde{\alpha}}, G_{\tilde{\rho}}\right)$ and $S\left(G_{\tilde{\rho}}, G_{\tilde{\pi}}\right)$ are reducible in $\mathcal{Q}^{(e)}$, then so is $S\left(G_{\tilde{\alpha}}, G_{\tilde{\pi}}\right)$. It is sufficient to show that all $S\left(G_{\tilde{\alpha}}, G_{\tilde{\rho}}\right)$ are reducible in $\mathcal{Q}^{(e)}$ for $\tilde{\alpha} \leq \tilde{\rho}$ componentwise.

We can further reduce our problem as follows. Assume that $\tilde{\alpha}$ and $\tilde{\rho}$ in $\mathcal{G}^{(e)}$ are generalized compositions of $d_{1}$ and $d_{2}$ respectively. If $\tilde{\alpha} \leq \tilde{\rho}$, then $d_{1} \leq d_{1}$. If $d_{2}-d_{1}>1$, we can select a generalized composition $\tilde{\alpha} \leq \tilde{\pi} \leq \tilde{\rho}$ and use (5.1) again. We can thus assume that $d_{2}-d_{1}=1$. That is, the two generalized compositions differ on one part by one unit.

Lemma 5.3. The set $\mathcal{Q}^{(e)}$ is a Gröbner basis of $\mathcal{J}^{(e)}$.

Proof. From the discussion above it is sufficient to show that all the expressions of $\mathcal{J}^{(e)}$ of the form $S\left(G_{\tilde{\gamma} a \tilde{\beta}}, G_{\tilde{\gamma}(a-1) \tilde{\beta}}\right)=G_{\tilde{\gamma} a \tilde{\beta}}-x_{k} G_{\tilde{\gamma}(a-1) \tilde{\beta}}$ where $k=\ell(\tilde{\gamma})+1$, are reducible in $\mathcal{Q}^{(e)}$. Let us denote by $m_{\tilde{\gamma} a \tilde{\beta}}\left(x_{1}, x_{2}, \ldots\right)$ the leading monomial of $S\left(G_{\tilde{\gamma} a \tilde{\beta}}, G_{\tilde{\gamma}(a-1) \tilde{\beta}}\right)$.

Let $\tilde{\beta}=\tilde{\nu} 00 \cdots$. We set up an induction on $\ell(\tilde{\nu})$. Assume first that $\tilde{\nu}=\nu$ is a (possibly empty) standard composition. The second part of Lemma 3.1 and the recursive definition (3.2) give $S\left(G_{\tilde{\gamma} a \nu 0 \ldots,}, G_{\tilde{\gamma}(a-1) \nu 0 \ldots)}=G_{\tilde{\gamma} 0 a \nu 0 \ldots \in \mathcal{Q}^{(e)}}\right.$. If 
$\tilde{\nu}$ is not standard, then let $\tilde{\beta}=\tilde{\pi} 0 b \mu 00 \cdots$ for $b>0$ and $\mu$ a (possibly empty) standard composition. Let $\ell=\ell(\tilde{\gamma} a \tilde{\pi})+1$. Using (3.2), we have

$$
\begin{aligned}
& S\left(G_{\tilde{\gamma} a \tilde{\pi} 0 b \mu 0 \ldots,} G_{\tilde{\gamma}(a-1) \tilde{\pi} 0 b \mu 0 \ldots)}\right)=G_{\tilde{\gamma} a \tilde{\pi} 0 b \mu 0 \ldots-x_{k} G_{\tilde{\gamma}(a-1) \tilde{\pi} 0 b \mu 0 \cdots}}
\end{aligned}
$$

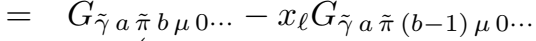

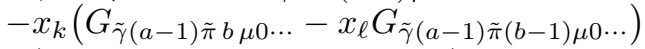

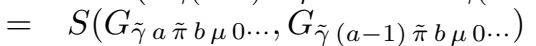

$$
\begin{aligned}
& -x_{\ell} S\left(G_{\tilde{\gamma} a \tilde{\pi}(b-1) \mu 0 \ldots,} G_{\tilde{\gamma}(a-1) \tilde{\pi}(b-1) \mu 0 \ldots) .}\right.
\end{aligned}
$$

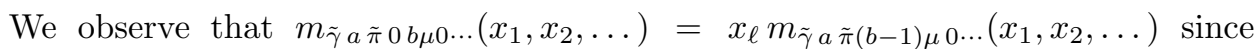
$m_{\tilde{\gamma} a \tilde{\pi} b \mu 0 \ldots\left(x_{1}, x_{2}, \ldots\right)}$ is distinct and lexicographically smaller than

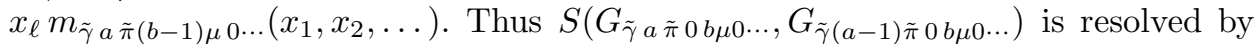
two expressions such that $\ell(\tilde{\pi} b \mu)=\ell(\tilde{\pi}(b-1) \mu)<\ell(\tilde{\nu})$ and by the induction hypothesis $S\left(G_{\tilde{\gamma} a \tilde{\pi} 0 b \mu 0 \ldots,} G_{\tilde{\gamma}(a-1) \tilde{\pi} 0 b \mu 0 \cdots)}\right)$ is reducible in $\mathcal{Q}^{(e)}$.

Corollary 5.4. The set $\mathcal{Q}^{(e)}$ is a Hilbert basis of $\overline{\mathcal{J}^{(e)}}$.

Proof. Given an element $P \in \overline{\mathcal{J}^{(e)}}$ let $X^{\tilde{\beta}}=L M(P)$. Since $\mathcal{Q}^{(e)}$ is a Gröbner basis it contains an element $G_{\tilde{\alpha}}$ such that $\tilde{\alpha} \leq \tilde{\beta}$ componentwise. Lemma 5.2 gives us that $G_{\tilde{\beta}} \in \mathcal{Q}^{(e)}$. The element $P-L C(P) \cdot G_{\tilde{\alpha}} \in \overline{\mathcal{J}^{(e)}}$ is such that $L M(P-L C(P)$. $\left.G_{\tilde{\alpha}}\right)<_{\text {lex }} X^{\tilde{\beta}}$. If we repeat this process (possibly countably many times) we can express $P$ as a series in the elements of $\mathcal{Q}^{(e)}$.

We are now in a position to conclude our investigation and prove the general version of our Theorem 1.1.

Corollary 5.5. The monomial Hilbert basis of $R^{(e)}$ is given by the monomials of $\mathbb{Q}\left[\left[x_{1}, x_{2}, x_{3}, \ldots\right]\right]$ corresponding to the paths of type e-Catalan.

Proof. As noted in Remark 3.5 above, the set $\left\{G_{\tilde{\alpha}}\right\}$ forms a Hilbert basis of $\mathbb{Q}\left[\left[x_{1}, x_{2}, x_{3}, \ldots\right]\right]$, and Corollary 5.4 gives that the set $\mathcal{Q}^{(e)}$ is a Hilbert basis of $\overline{\mathcal{J}^{(e)}}$. Thus a Hilbert basis of the quotient $R^{(e)}=\mathbb{Q}\left[\left[x_{1}, x_{2}, x_{3}, \ldots\right]\right] / \overline{\mathcal{J}^{(e)}}$ is given by the set $\left\{G_{\tilde{\alpha}}\right\} \backslash \mathcal{Q}^{(e)}=\left\{G_{\tilde{\alpha}} \mid \tilde{\alpha}\right.$ corresponds to a path of type e-Catalan $\}$. The result follows by triangularity.

Remark 5.6. To show the equality in Theorem 1.2, it appears that the set $\left\{G_{\tilde{\alpha}} \mid\right.$ $\ell(\tilde{\alpha})=n$, and $\tilde{\alpha}$ reaches level $e\}$ forms a linear basis of $J_{n}^{(e)}$. Unfortunately the argument of Section 5 is not sufficient to show this with finitely many variables and it requires more work. This is the object of our collaboration in 2 .

\section{ACKNOWLEDGMENTS}

We are indebted to François Bergeron and Adriano Garsia for extremely stimulating conversations and valuable suggestions. We also thank Mike Zabrocki and Geanina Tudose for ideas and comments.

\section{REFERENCES}

[1] E. Artin, Galois Theory, Notre Dame Mathematical Lecture 2 (1944), Notre Dame, IN. MR 5:225c reprint MR 98k:12001 MR 42:234

[2] J.-C. Aval, F. Bergeron and N. Bergeron, Ideal of quasi-symmetric functions and supercovariant polynomials for $s_{n}$, to appear (Math. co 10202071).

[3] J.-C. Aval, F. Bergeron, N. Bergeron and A. Garsia, Ideals of Quasi-symmetric polynomials and related varieties, in preparation. 
[4] F. Bergeron, N. Bergeron, A. Garsia, M. Haiman and G. Tesler, Lattice Diagram Polynomials and Extended Pieri Rules, Adv. Math. 142 (1999), 244-334. MR 2000h:05231

[5] F. Bergeron, A. Garsia and G. Tesler, Multiple Left Regular Representations Generated by Alternants, J. of Comb. Th., Series A 91, 1-2 (2000), 49-83. MR 2002c:05158

[6] N. Bergeron, S. Mykytiuk, F. Sottile, and S. van Willigenburg, Pieri Operations on Posets, J. of Comb. Theory, Series A 91 (2000), 84-110.

[7] C. DE Concini And C. Procesi, Symmetric functions, conjugacy classes and the flag variety, Invent. Math. 64 (1981), 203-230. MR 82m:14030

[8] D. Cox, J. Little And D. O'Shea, Ideals, Varieties, and Algorithms, Springer-Verlag, NewYork, 1992. MR 93j:13031; second edition MR 97h:13024

[9] A. M. Garsia and M. Haiman, A graded representation model for Macdonald's polynomials, Proc. Nat. Acad. Sci. U.S.A. 90 (1993), no. 8, 3607-3610. MR 94b:05206

[10] I. Gelfand, D. Krob, A. Lascoux, B. Leclerc, V. Retakh, and J.-Y. Thibon, Noncommutative symmetric functions, Adv. in Math. 112 (1995), 218-348. MR 96e:05175

[11] I. Gessel, Multipartite P-partitions and products of skew Schur functions, in Combinatorics and Algebra (Boulder, Colo., 1983), C. Greene, ed., vol. 34 of Contemp. Math., AMS, 1984, pp. 289-317. MR 86k:05007

[12] M. Haiman, Hilbert schemes, polygraphs, and the Macdonald positivity conjecture, J. Amer. Math. Soc. 14 (2001), 941-1006. MR 2002c:14008

[13] F. HIVERT, Hecke algebras, difference operators, and quasi-symmetric functions, Adv. Math. 155 (2000), 181-238. CMP 2001:04

[14] I. Macdonald, Symmetric Functions and Hall Polynomials, Oxford Univ. Press, 1995, second edition. MR 96h:05207

[15] C. Malvenuto and C. Reutenauer, Duality between quasi-symmetric functions and the Solomon descent algebra, Journal of Algebra 177 (1995), 967-982. MR 97d:05277

[16] R. Stanley, Enumerative Combinatorics, Vol. 1, Wadsworth and Brooks/Cole, 1986. MR 87j:05003 corr. reprint MR 98a:05001

[17] R. Stanley, Enumerative Combinatorics Vol. 2, no. 62 in Cambridge Studies in Advanced Mathematics, Cambridge University Press, 1999. Appendix 1 by Sergey Fomin. MR 2000k:05026

[18] R. Steinberg, Differential equations invariant under finite reflection groups, Trans. Amer. Math. Soc. 112 (1964), 392-400. MR 29:4807

Laboratoire A2X, Université Bordeaux 1, 351 cours de la libération, 33405 Talence CEDEX, FRANCE

E-mail address: aval@math.u-bordeaux.fr

Department of Mathematics and Statistics, York University, Toronto, Ontario, CANADA M3J 1P3

E-mail address: bergeron@mathstat.yorku.ca

$U R L:$ http://www. math. yorku.ca/bergeron 